\title{
Topologically Massive AdS Gravity
}

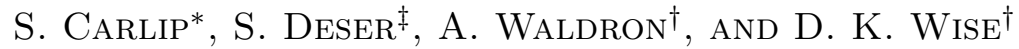 \\ * Department of Physics, University of California, Davis, CA 95616, USA \\ carlip@physics.ucdavis.edu \\ ${ }^{\ddagger}$ California Institute of Technology, Pasadena, CA 91125 and \\ Brandeis University, Waltham, MA 02454, USA \\ deser@brandeis.edu \\ ${ }^{\dagger}$ Department of Mathematics, University of California, Davis, CA 95616, USA \\ wally, derek@math .ucdavis.edu
}

\begin{abstract}
We analyze $(2+1)$-dimensional gravity with a Chern-Simons term and a negative cosmological constant, primarily at the weak field level. The full theory is expressible as the sum of two higher derivative $\mathrm{SL}(2, \mathbb{R})$ "vector" ChernSimons terms, while the physical bulk degrees of freedom correspond to a single massive scalar field, just as for $\Lambda=0$. The interplay of $\Lambda$ and the mass parameter $\mu$ can be studied, and any physical mass - including the conformal value with null propagation - is accessible by tuning $\mu$. The single bulk mode yields a complete set of normalizable positive energy wave packets, as long as one chooses the usual, "wrong" sign of $G$ necessary to connect smoothly with the known $\Lambda=0$ limit. The chiral Chern-Simons coupling leads to gauge invariant linearized curvatures propagating with chirality-dependent masses. Linearized metric fluctuations have a finite asymptotic Fefferman-Graham expansion about the Poincaré metric for any mass value greater or equal to a "critical" one, where various amusing effects appear, such as vanishing of one of the two "vector" Chern-Simons terms and an equivalence between tensor and vector excitations. We also find a set of chiral, pp-wave metrics that exactly solve the full nonlinear equations.
\end{abstract}

\section{Introduction}

We report some novel properties of a currently popular extension [1-5] of $(2+1)$ dimensional topologically massive gauge theories [6]. The latter are described by vector (Abelian or Yang-Mills) or tensor (Einstein) actions supplemented by ChernSimons terms, while the generalizations adjoin an anti-de Sitter (AdS) background for vectors and a cosmological constant for gravity. Just as for four-dimensional 
massive tensor models $[7,8]$, the $\left(m^{2}, \Lambda\right)$ plane displays unexpected richness, beyond the surprises already found in the $\Lambda=0$ models [6]. The technology is sufficiently involved that we only provide and motivate some highlights of the emergent properties, and do so primarily for weak field excitations of gravity about the AdS vacuum. Details may be found in [9].

Two effects are particularly striking. The first is that although there is one bulk excitation for all mass values, suitable tuning can yield null propagation at finite mass, even without new gauge invariances of the sort that appear for massive higher spins in higher dimensions $[7,8]$. Even more surprisingly, the various curvature components of this excitation propagate with different, chirality-dependent masses.

We shall first survey scalars and vectors in $\mathrm{AdS}_{3}$, showing that a vector field has a single, gauge-invariant, massive bulk mode. We display the chirality-dependent masses of the individual field strength components, and show how $\left(m^{2}, \Lambda\right)$ tuning can effect null cone propagation. We next analyze the more complex gravity model, which displays the same pattern: a single physical gauge-invariant "scalar" mode whose corresponding curvature components carry chirality-dependent masses. The bulk excitations consist of a complete set of localized wave packets, all with positive energy (with the same overall sign choice as for $\Lambda=0$ ) and good asymptotic behavior. Separately, there are exact pp-wave chiral solutions. Both sets of solutions are present for all masses, modulo some fine print below certain mass bounds. Finally, we cast our model into "pure Chern-Simons" form and note some formal properties at critical mass values.

\section{$2 \quad \mathrm{AdS}_{3}$ and Scalars}

We denote the AdS metric $g_{\mu \nu}$ and the full dynamical metric $\gamma_{\mu \nu}$, with signature $(-++)$. In our conventions, the Ricci tensor is $R_{\mu \nu}=\partial_{\rho} \Gamma_{\mu \nu}^{\rho}+\cdots=2 \Lambda g_{\mu \nu}$; the Riemann tensor is its double dual. We choose units $\Lambda=-1$. The Poincaré patch,

$$
d s^{2}=g_{\mu \nu} d x^{\mu} d x^{\nu}=\left(2 d x^{+} d x^{-}+d z^{2}\right) / z^{2},
$$

covers only part of AdS, but the remainder is easily reached by inversions. We often make the lightlike time choice $\tau=x^{+}$.

Consider first a standard (positive-energy) massive scalar, whose unimproved action reduces to

$$
I=\int d \tau\left\{\langle\phi, \dot{\phi}\rangle-\left(\frac{1}{2}\left[\partial_{z} \phi\right]^{2}+\frac{1}{2 z^{2}}\left[m^{2}+\frac{3}{4}\right] \phi^{2}\right) d x^{-} d z\right\} .
$$

Here we have performed the field redefinition $\phi \equiv \frac{1}{\sqrt{z}} \varphi$, where $\varphi$ is the usual scalar field; the symplectic current is defined by the antisymmetric bracket $\langle A, B\rangle=$ $\int d x^{-} d z A \partial_{-} B$. With the above conventions, the field propagates according to

$$
\left[2 \partial_{-} \partial_{+}+\partial_{z}^{2}-\frac{m^{2}+3 / 4}{z^{2}}\right] \phi=0 \text {. }
$$


At $m^{2}=-3 / 4$, this is the Minkowski wave equation; indeed, this mass corresponds to adding the usual conformal improvement term $\frac{1}{16} R \varphi^{2}$. Masses as negative as $m^{2}=-1$ are permitted, that being the well-known Breitenlohner-Freedman bound [10]. A Fourier transform on $\partial_{ \pm}$to $\omega^{2}=E^{2}-p_{x}^{2}$ yields the Bessel equation

$$
\left[\frac{d^{2}}{d z^{2}}+\frac{1}{z} \frac{d}{d z}+\omega^{2}-\frac{\nu^{2}}{z^{2}}\right]\left(\frac{\varphi}{z}\right)=0, \quad \nu^{2}=m^{2}+1 .
$$

Its solutions oscillate, and at half-integer values of $\nu$ they become the product of a slowly varying function times a Minkowski plane wave $\exp (i k \cdot x)$ with $k^{2}=0$, giving quasi-lightlike propagation. Indeed, the lowest value, $\nu^{2}=(1 / 2)^{2}$, is the "improved" massless scalar. In the singular case $\omega=0$, i.e., $\partial_{ \pm} \phi=0$, chiral solutions appear; when viewed as metric fluctuations, they are pp-waves, as we discuss later. Some facts regarding the bulk solutions in our patch, whose boundary includes both $z=0$ and a line at $z=\infty$ : finiteness at $z=0$ is achieved by choosing the Bessel function solution $J_{\nu} \sim z^{\nu}$ at the origin; to achieve finiteness at the other end requires choosing bounded wave packets, which we describe below.

\section{Vectors}

The Abelian vector system has an action

$$
I=-\frac{1}{4} \int d^{3} x\left\{\sqrt{-g} F_{\mu \nu} g^{\mu \rho} g^{\nu \sigma} F_{\rho \sigma}+\mu \varepsilon^{\mu \nu \rho} F_{\mu \nu} A_{\rho}\right\}
$$

where $\mu$ has the dimension of mass. Note that the Chern-Simons term is metricindependent. The model has a single, gauge-invariant, "scalar" component

$$
\phi=\sqrt{z}\left[A_{z}-\left(\partial_{z} / \partial_{-}\right) A_{-}\right]=\sqrt{z} \partial_{-}^{-1} F_{-z},
$$

assuming invertibility of $\partial_{-}$. Observe that since $\phi$ is gauge-invariant, the analysis simplifies by using light-front gauge $A_{-}=0$; this lesson is especially useful when we move on to the tensor case. This $\phi$ obeys the scalar equation (3), but with a shifted mass, $\nu^{2}=m^{2}+1=(\mu+1)^{2}$. All values of $\mu$ obey the $m^{2} \geq-1$ bound. Notably, the value required for lightlike propagation occurs at half-integer $\nu$ rather than at $\mu=0$.

Now consider the three components $\left(F_{+z}, F_{+-}, F_{-z}\right)$ of the field strength. Here we find a surprise: although the components come from a single "scalar" mode, they propagate according to scalar wave equations with different masses,

$$
m^{2}=\left((\mu-1)^{2}-1, \mu^{2}-1,(\mu+1)^{2}-1\right) .
$$

This result is manifestly symmetric under simultaneous $\mu$ and helicity flips. Finally, it is clear that the energy remains positive in AdS: since the vector Chern-Simons term is purely topological, it does not contribute to the stress-energy tensor, which differs from its flat value only by an overall $z^{2}$ factor coming from the metric (1). 


\section{Gravitons}

The complete action for our model, in terms of the dynamical metric $\gamma_{\mu \nu}$, is

$$
I=\int d^{3} x\left\{-\sqrt{-\gamma}(R-2 \Lambda)+\frac{1}{2 \mu} \varepsilon^{\mu \nu \rho}\left(\Gamma_{\mu \beta}^{\alpha} \partial_{\nu} \Gamma_{\rho \alpha}^{\beta}+\frac{2}{3} \Gamma_{\mu \gamma}^{\alpha} \Gamma_{\nu \beta}^{\gamma} \Gamma_{\rho \alpha}^{\beta}\right)\right\} .
$$

As in the $\Lambda=0$ model, the sign of the Einstein part is "wrong," opposite to that in four dimensions. Writing $\gamma_{\mu \nu}=g_{\mu \nu}+h_{\mu \nu}$, we find that physical quantities can be expressed at first order in terms of the linearized-diffeomorphism-invariant "scalar"

$$
\varphi \equiv h_{z z}-2\left(\left[\partial_{z}+\frac{1}{z}\right] / \partial_{-}\right) h_{-z}+\left(\left[\partial_{z}+\frac{1}{z}\right]\left[\partial_{z}+\frac{2}{z}\right] / \partial_{-}^{2}\right) h_{--} .
$$

This reduces to $h_{z z}$ in light front gauge $h_{\mu-}=0$, where the metric becomes

$$
d s^{2}=\frac{2 d x^{+} d x^{-}+d z^{2}}{z^{2}}+\left(d x^{+}\right)^{2} h_{++}+2 d x^{+} d z h_{z+}+d z^{2} h_{z z}+\mathcal{O}\left(h^{2}\right) .
$$

Defining $\phi=z^{3 / 2} h_{z z}$, we again find the scalar action (2), but now with mass

$$
m^{2}=(\mu+2)^{2}-1 .
$$

The analog of the "field strength" is the linearized cosmological Einstein tensor,

$$
\mathcal{H}_{\rho \sigma}=\left[G_{\rho \sigma}-g_{\rho \sigma}\right]_{\text {LINEAR }},
$$

which is invariant under linearized diffeomorphisms. As in the vector case, the components propagate with different masses: $\left(\mathcal{H}_{++}, \mathcal{H}_{+z}, \mathcal{H}_{z z}=-2 \mathcal{H}_{+-}, \mathcal{H}_{z-}, \mathcal{H}_{--}\right)$ have masses

$$
m^{2}=\left((\mu-2)^{2}-1,(\mu-1)^{2}-1, \mu^{2}-1,(\mu+1)^{2}-1,(\mu+2)^{2}-1\right) .
$$

The results are again invariant under simultaneous $\mu$ and chirality flips $\mu \leftrightarrow-\mu$, $+\leftrightarrow-$.

All values of $\mu$ respect the $m^{2} \geq-1$ bound, so the excitations represent a consistent positive energy system. Moreover, the Bianchi identities guarantee that every $\mathcal{H}_{\mu \nu}$ solution comes from a linearized metric fluctuation. We must still, however, check the asymptotic behavior of the perturbed geometry. It can be shown that the appropriate Bessel function solutions of (4) lead to metrics that are asymptotically AdS at $z=0$, with finite curvature invariants and finite contractions of $\mathcal{H}_{\mu \nu}$ with AdS unit vectors. If we demand the stronger Fefferman-Graham [11] conditions of finite $h_{\mu \nu}$ at $z=0$, on the other hand, we find a restriction $\mu \geq 1$.

More problematic is the large $z$ behavior, where $J_{\nu} \sim z^{-1 / 2}$, with a consequent divergence of $\mathcal{H}_{\mu \nu} \sim z^{+1 / 2}$ for all $\mu$. This means a breakdown at the $z \rightarrow \infty$ piece of the boundary of our Poincaré patch. Fortunately, this difficulty is circumvented 
by a complete, normalizable set of bounded wave packets that avoid this edge. This set exists for all $\mu$, including the $\mu=1$ "critical" value, so a physical bulk degree of freedom is always present, as we now show.

The $h_{z z}$ modes are

$$
\begin{aligned}
h_{\omega k}(x, z, t) & =\sqrt{\frac{\omega}{4 \pi E}} \frac{1}{z} J_{\mu+2}(\omega z) e^{i k x-i E t}, \\
h_{\omega k}^{*}(x, z, t) & =\sqrt{\frac{\omega}{4 \pi E}} \frac{1}{z} J_{\mu+2}(\omega z) e^{-i k x+i E t},
\end{aligned}
$$

with $E=\sqrt{\omega^{2}+k^{2}}$. A conserved bilinear current $\mathcal{J}^{\mu}=z^{4} g^{\mu \nu}\left(h_{1} \partial_{\nu} h_{2}-[1 \leftrightarrow 2]\right)$ defines a time-independent Klein-Gordon inner product $(\cdot, \cdot)$ with respect to which these modes are (continuum) orthonormal:

$$
\left(h_{\omega k}, h_{\omega^{\prime} k^{\prime}}\right)=-\left(h_{\omega k}^{*}, h_{\omega^{\prime} k^{\prime}}^{*}\right)=\delta\left(\omega-\omega^{\prime}\right) \delta\left(k-k^{\prime}\right), \quad\left(h_{\omega k}, h_{\omega^{\prime} k^{\prime}}^{*}\right)=0 .
$$

Arbitrary superpositions

$$
h_{z z}(x, z, t)=\int d \omega d k\left[a(\omega, k) h_{\omega k}(x, z, t)+a^{*}(\omega, k) h_{\omega k}^{*}(x, z, t)\right]
$$

provide general solutions with support bounded away from $z=\infty$. The norm

$$
(h, h)=\int d \omega d k\left[a(\omega, k)^{2}-a^{*}(\omega, k)^{2}\right]
$$

is constant in time and of the standard Klein-Gordon form, with the usual sign for the negative frequency modes.

The conserved energy of the excitations can now be evaluated, giving

$$
\begin{aligned}
H & =\frac{1}{2} \int d x d x z^{3}\left[\left(\partial_{t} h\right)^{2}+\left(\partial_{x} h\right)^{2}+\left(\partial_{z} h\right)^{2}+\frac{(\mu+2)^{2}-1}{z^{2}} h^{2}\right] \\
& =\frac{1}{2} \int d x d x z^{3}\left[h \partial_{t}^{2} h-\left(\partial_{t} h\right)^{2}\right]=\int d \omega d k \sqrt{k^{2}+\omega^{2}}|a(\omega, k)|^{2},
\end{aligned}
$$

upon (permitted) integrations by parts. Finite energy at an initial time then guarantees asymptotically AdS behavior at the entire boundary for all times. These considerations are also valid for $\mu=1$, the point at which it was claimed in [4] that "massive gravitons" disappear. The authors of [4] considered only eigenfunctions of the compact $\mathrm{SL}(2, \mathbb{R})$ generator; our wave packet solutions, while not such eigenstates, are nevertheless a perfectly acceptable complete set of functions (also for $\mu=1$ ). They require the "wrong" sign choice in (17) for positive energy. 


\section{Chern-Simons Formulations}

Amusingly, our model (7), in dreibein $e_{\mu}{ }^{a}$ rather than metric variables, can be cast in "pure," but higher derivative, Chern-Simons form

$$
\left.I_{\mathrm{TMG}}[e]=-\frac{1}{2}\left(1-\frac{1}{\mu}\right) I^{+} A[e]\right]+\frac{1}{2}\left(1+\frac{1}{\mu}\right) I[-A[e]],
$$

where

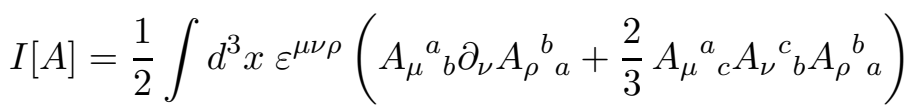

and

$$
{ }^{ \pm} A_{\mu}{ }^{a} b[e]=\omega_{\mu}{ }^{a}[e] \pm \varepsilon^{a}{ }_{b c} e_{\mu}{ }^{c} .
$$

Here $\omega[e]$ is the usual torsion-free spin connection, and the coefficients of the two Chern-Simons terms are the left/right central charges. In particular, at $\mu= \pm 1$ the action reduces to a single term (R. Jackiw and D. Grumiller have also observed this-private communication). The scheme is reminiscent of the earlier ATW [12] formulation of cosmological Einstein gravity, but differs because of the higher derivatives in the gravitational Chern-Simons term. Our model's propagating modes are due to these higher derivatives, as has long been understood [13]. A canonical formulation of the full theory (17), extending the $\Lambda=0$ analysis of [14], also sets to rest any issues regarding the excitation content for all $\mu$. The results of our linearized analysis have recently been confirmed in [15] at $\mu=1$, and an independent computation [16] at arbitrary $\mu$ gives the same result: a single bulk degree of freedom occurs for all $|\mu| \in(0, \infty)$.

In addition to the reduction from two Chern-Simons terms to one at $\mu=1$, a quite different special result occurs at this value: linearized topologically massive

gravity becomes equivalent to topologically massive electrodynamics. Solutions of the latter determine all gravity solutions through the equivalence

$$
\mathcal{H}_{\mu \nu}=D_{(\mu} F_{\nu)},
$$

where $F_{\nu}$ is the dual Maxwell field strength and $D_{\mu}$ is the AdS covariant derivative.

\section{Chiral pp-Waves}

We noted earlier that in addition to the (non-chiral) bulk modes, there exist chiral ones; we sketch them here. In contrast to the wave packets discussed above, these chiral states are in fact solutions of the full nonlinear field equations. The ansatz

$$
d s^{2}=\frac{2 d x^{+}\left(d x^{-}+z^{2+\gamma} h\left(x^{+}\right) d x^{+}\right)+d z^{2}}{z^{2}}
$$

solves the nonlinear field equations for the three values $\gamma=(-2,0, \mu-1)$. The first two are also solutions of pure Einstein gravity, and of these only $\gamma=0$ is 
asymptotically well-behaved. The third solution is a chiral pp-wave for all values of $\mu \neq \pm 1$, with finite asymptotic behavior for $\mu>1$. At $\mu=1$, the ansatz (21) must be replaced by one logarithmic in $z$, which is asymptotically AdS but not finite at the boundary in the Fefferman-Graham sense.

In this connection, omitting details, it is worth giving a representation-theoretic description of the equations and solutions in terms of the $\mathrm{SL}(2, \mathbb{R}) \times \mathrm{SL}(2, \mathbb{R}) \mathrm{AdS}_{3}$ isometry group, with Killing vectors

$$
\begin{aligned}
L_{+} & =\partial_{+}, & R_{+} & =\partial_{-}, \\
L_{0} & =-x^{+} \partial_{+}-\frac{1}{2} z \partial, & R_{0} & =-x^{-} \partial_{-}-\frac{1}{2} z \partial, \\
L_{-} & =-x^{+}\left(x^{+} \partial_{+}+z \partial\right)+\frac{1}{2} z^{2} \partial_{-}, & R_{-} & =-x^{-}\left(x^{-} \partial_{-}+z \partial\right)+\frac{1}{2} z^{2} \partial_{+},
\end{aligned}
$$

and equal left/right Casimirs related to the scalar Laplacian by

$$
\Delta_{L / R}=\left\{L_{+}, L_{-}\right\}+2 L_{0}^{2}=\left\{R_{+}, R_{-}\right\}+2 R_{0}^{2}=\frac{1}{2} z^{2}\left(2 \partial_{+} \partial_{-}+z \partial \frac{1}{z} \partial\right)=\frac{1}{2} \Delta .
$$

The bulk solutions we have discussed so far are not chiral, and the bulk-boundary propagator is the intertwiner between the reducible representation of the isometry group generated by the Killing vectors and irreducible quasiprimary boundary fields. The additional chiral pp-wave solutions are obtained by requiring that $L_{+}$and $R_{+}$ annihilate the highest chirality curvature component $\mathcal{H}_{--}$and that $R_{+}$annihilate all other curvature fluctuations. In this case one finds discrete series representations for one of the $\mathrm{SL}(2, \mathbb{R})$ factors and a singlet for the other, and therefore a chiral subsector of the theory.

\section{Conclusions}

We have surveyed some of the novel features of cosmologically extended topologically massive gravity, as well as those that reduce smoothly in the $\Lambda \rightarrow 0$ limit. Among the latter is the existence of a single massive spin 2 excitation for all $\mu$, which has positive energy as long as the same "wrong" sign Einstein term is used as in the $\Lambda=0$ case. The weak field solutions consist of a complete set of non-chiral wave packets, along with pp-wave chiral solutions. Among the novelties associated with the $\left(m^{2}, \Lambda\right)$ plane, we found that the curvature excitations propagate with masses that are both shifted and internally split according to chirality; and that tunings exist allowing for null propagation at special mass values. We also found a two-term "pure Chern-Simons" expression for our action in terms of a composite, higher-derivative sum of torsionless spin connection and dreibein fields; at $\mu= \pm 1$,

one of these two terms vanishes. Separately, we formulated an explicit equivalence between vector and tensor excitations at $\mu=1$. 
Finally, we mention the possible stability issue posed by the BTZ black hole, which is also an exact solution to our model. With the "wrong" sign choice required to keep the bulk mode stable, the black hole has a negative mass. Classically, however, it is not clear that negative energy black holes can be created from positive energy matter or source-free excitations, and at the quantum level, we know of no instanton that mediates their creation. Indeed, there may well be a superselection argument that excludes the BTZ metric. One strong hint in this direction comes from the fact that the model has a supergravity extension (for $\Lambda<0$, as usual) which is necessarily of positive energy by the usual $H=\left\{Q, Q^{*}\right\}>0$ relation [17].

\section{Acknowledgments}

We thank D. Grumiller, R. Jackiw, N. Johansson, W. Li, D. Marolf, W. Song, and A. Strominger for discussions. This work was supported by the National Science Foundation under grants PHY07-57190 and DMS-0636297 and the Department of Energy under grants DE-FG02-91ER40674 and DE-FG02-92-ER40701.

\section{References}

[1] S. Deser and B. Tekin, "Energy in topologically massive gravity," Class. Quant. Grav. 20, L259 (2003); S. Deser and B. Tekin, "Massive, topologically massive, models," Class. Quant. Grav. 19, L97 (2002) arXiv:hep-th/0203273.

[2] P. Kraus and F. Larsen, "Holographic gravitational anomalies," JHEP 0601, 022 (2006) arXiv:hep-th/0508218].

[3] S. N. Solodukhin, "Holography with gravitational Chern-Simons term," Phys. Rev. D74, 024015 (2006) arXiv:hep-th/0509148.

[4] W. Li, W. Song, and A. Strominger, "Chiral gravity in three dimensions" arXiv:0801.4566v] (hep-th); "Comment on 'Cosmological Topological Massive Gravitons and Photons'," arXiv:0805.3101 [hep-th].

[5] D. Grumiller and N. Johansson, "Instability in cosmological topologically massive gravity at the chiral point," arXiv:0805.2610 [hep-th].

[6] S. Deser, R. Jackiw and S. Templeton, "Three-dimensional massive gauge theories," Phys. Rev. Lett. 48, 975 (1982); "Topologically massive gauge theories," Annals Phys. 140, 372 (1982).

[7] S. Deser and R. I. Nepomechie, "Anomalous propagation of gauge fields in conformally flat spaces," Phys. Lett. B 132, 321 (1983); "Gauge invariance versus masslessness in de Sitter space," Annals Phys. 154, 396 (1984). 
[8] S. Deser and A. Waldron, "Gauge invariances and phases of massive higher spins in (A)dS," Phys. Rev. Lett. 87, 031601 (2001) arXiv:hep-th/0102166; "Partial masslessness of higher spins in (A)dS," Nucl. Phys. B 607, 577 (2001) arXiv:hep-th/0103198; "Stability of massive cosmological gravitons," Phys. Lett. B 508, 347 (2001) |arXiv:hep-th/0103255]; "Null propagation of partially massless higher spins in (A)dS and cosmological constant speculations," Phys. Lett. B 513, 137 (2001) arXiv:hep-th/0105181.

[9] S. Carlip, S. Deser, A. Waldron and D. K. Wise, "Cosmological Topologically Massive Gravitons and Photons," arXiv:0803.3998 [hep-th].

[10] P. Breitenlohner and D. Z. Freedman, "Stability in gauged extended supergravity," Annals Phys. 144, 249 (1982); "Positive energy in anti-de Sitter backgrounds and gauged extended supergravity," Phys. Lett. B 115, 197 (1982). L. Mezincescu and P. K. Townsend, "Stability at a local maximum in higher dimensional anti-de Sitter space and applications to supergravity," Annals Phys. 160, 406 (1985).

[11] C. Fefferman and C.R. Graham, "Conformal invariants," Elie Cartan et les Mathematiques d'Aujourdhui (Asterique, 1985) 95.

[12] A. Achúcarro and P.K. Townsend, "A Chern-Simons action for threedimensional anti-de Sitter supergravity theories", Phys. Lett. B 180, 89 (1986); E. Witten, "(2+1)-dimensional gravity as an exactly soluble system," Nucl. Phys. B 311, 46 (1988).

[13] S. Deser and R. Jackiw, "Higher derivative Chern-Simons extensions," Phys. Lett. B 451, 73 (1999) arXiv:hep-th/9901125.

[14] S. Deser and X. Xiang, "Canonical formulations of full nonlinear topologically massive gravity," Phys. Lett. B 263, 39 (1991).

[15] D. Grumiller, R. Jackiw and N. Johansson, "Canonical analysis of cosmological topologically massive gravity at the chiral point," arXiv:0806.4185 [hep-th].

[16] S. Carlip, "The Constraint Algebra of Topologically Massive AdS Gravity", to appear.

[17] S. Deser, "Cosmological topological supergravity," in Quantum Theory of Gravity, edited by S. M. Christensen, Adam Hilger, London, 1984; S. Deser, "Massive spin 3/2 theories in three-dimensions," Phys. Lett. B 140, 321 (1984). 